\title{
Bicolored Matchings in Some Classes of Graphs
}

\author{
M. C. Costa ${ }^{1}$, D. de Werra ${ }^{2}$, C. Picouleau ${ }^{1}$, and B. Ries ${ }^{2}$ \\ ${ }^{1}$ CEDRIC, CNAM, Paris. e-mail: costa@cnam.fr,chp@cnam.fr \\ 2 IMA - EPFL, Lausanne. e-mail: dewerra.ima@epfl.ch,bernard.ries@epfl.ch
}

\begin{abstract}
We consider the problem of finding in a graph a set $R$ of edges to be colored in red so that there are maximum matchings having some prescribed numbers of red edges. For regular bipartite graphs with $n$ nodes on each side, we give sufficient conditions for the existence of a set $R$ with $|R|=n+1$ such that perfect matchings with $k$ red edges exist for all $k, 0 \leq k \leq n$. Given two integers $p<q$ we also determine the minimum cardinality of a set $R$ of red edges such that there are perfect matchings with $p$ red edges and with $q$ red edges. For 3-regular bipartite graphs, we show that if $p \leq 4$ there is a set $R$ with $|R|=p$ for which perfect matchings $M_{k}$ exist with $\left|M_{k} \cap R\right| \leq k$ for all $k \leq p$. For trees we design a linear time algorithm to determine a minimum set $R$ of red edges such that there exist maximum matchings with $k$ red edges for the largest possible number of values of $k$.
\end{abstract}

Key words. Matchings, Alternating cycles, Bicolored graphs, Cacti, bipartite graphs, Lineperfect graphs, trees

\section{Introduction}

Various types of packing problems in graphs have been extensively studied by many authors; the maximum stable set problem (find a maximum cardinality set of mutually non adjacent nodes); the maximum matching problem (find a maximum cardinality set of mutually non adjacent edges) and the maximal forest problem are some of the most famous examples (see for instance [3] for a formulation of many basic packing problems in graph theory).

A natural extension of packing problems has been considered in several forms. It consists in giving a bicoloring $(R, B)$ (for red and black) of the node set (resp. of the edge set) of a graph $G=(V, E)$; it is then required to find if $G$ contains a stable set $S$ (resp. a matching $M$ ) such that $|S \cap R| \geq p$ and $|S \cap B| \geq q$ (resp. $|M \cap R| \geq p,|M \cap B| \geq q$ ) where $p$ and $q$ are given positive integers (see [7, 15]).

Besides this, the problem of constructing a spanning tree $T$ in a graph $G=(V, E)$ whose edge set is partitioned into sets $R, B$ is considered in [7] with the requirement that $|T \cap R| \geq p$ : it is shown that a solution can easily be constructed by using simple adaptations of basic algorithms.

In addition, the problem of constructing a bicolored perfect matching $M$ in a complete bipartite graph $K_{n, n}$ (both the left set and the right set consist of exactly $n$ nodes) is considered in [15] with the requirement that $|M \cap R|=p$; it is a special case 
of the problem consisting of determining whether in a complete bipartite graph $K_{n, n}$ where each edge [i,j] has a weight $w_{i j}$ there exists a perfect matching $M$ with weight $w(M) \equiv \sum\left(w_{i j} \mid[i, j] \in M\right)=p$. This general case was shown to be NP-hard in [4], while the special case $w_{i j}=1$ if $[i, j] \in R$ and $w_{i j}=0$ else was solved with a polynomial algorithm in $[11,15]$. The complexity of the problem with 0,1 -weights in general bipartite graphs is apparently unknown.

In this paper we intend to consider a related problem which is also based on a bicoloring $(R, B)$ of the edge set of a graph. We will essentially try to characterize "minimal" sets $R$ for which maximum matchings $M$ can be found with specific values of $p=|M \cap R|$.

More specifically, given a graph $G=(V, E)$ and a set $\mathcal{P}=\left\{p_{0}, p_{1}, \ldots, p_{s}\right\}$ of integers $0 \leq p_{0}<p_{1}<\ldots<p_{s} \leq\lfloor|V| / 2\rfloor$, we want to color a subset $R \subseteq E$ of edges of $G$, say in red, in such a way that for any $i(0 \leq i \leq s) G$ contains a maximum matching $M_{i}$ with exactly $p_{i}$ red edges, i.e., $\left|M_{i} \cap R\right|=p_{i}$.

We shall in particular be interested in finding a smallest subset $R$ for which the required maximum matchings do exist.

A subset $R$ will be $\mathcal{P}-$ feasible for $G$ if for every $p_{i}$ in $\mathcal{P}$ there is a maximum matching $M_{i}$ in $G$ with $\left|M_{i} \cap R\right|=p_{i}$. Notice that for some $\mathcal{P}$ there may be no $\mathcal{P}$-feasible set $R$ (take $\mathcal{P}=\{0,1,2\}$ in $G=K_{2,2}$ ).

In section 2 we will derive some elementary properties of solutions in regular bipartite graphs. Section 3 will be devoted to a special case where the set of values $p=|M \cap R|$ is an interval of consecutive integers. Then section 4 will be dedicated to a linear algorithm for trees. Finally section 5 will contain conclusions and possible extensions.

All graph theoretical terms not defined here can be found in [3]. Basic properties of matchings are to be found in [12]. For definitions linked to complexity, the reader is referred to [13]. In general all graphs will be simple (no multiple edges, no loops).

\section{Regular Bipartite Graphs}

In this section we will state some basic results concerning $\mathcal{P}$-feasible sets in regular bipartite graphs.

Proposition 1. In a $\triangle$-regular bipartite graph $G$ for any $\mathcal{P}$ with $|\mathcal{P}| \leq \Delta$ there exists a $\mathcal{P}$-feasible set $R$.

This follows from the fact that the edge set of $G$ can be partitioned into $\triangle$ perfect (and hence maximum) matchings (by the König theorem, see [3], chapter 12).

Let us now briefly consider a special case for a $\triangle$-regular bipartite graph.

Theorem 1. Let $G=(X, Y, E)$ with $|X|=|Y|=n$ be a $\triangle$-regular bipartite graph, with $\triangle \geq 2$, and let $\mathcal{P}=\{p, q\}$ with $1 \leq p<q \leq n$. The minimum cardinality of $a$ $\mathcal{P}$-feasible set $R$ is given by

$$
|R|=q+\max \{0, p-n+|\mathcal{C}| / 2\}
$$


where $\mathcal{C}$ is a collection of node disjoint cycles which are alternating with respect to a perfect matching and which have a minimum total length $|\mathcal{C}|=\sum_{C_{i} \in \mathcal{C}}\left|C_{i}\right|$ satisfying $|\mathcal{C}| / 2 \geq q-p$.

Proof. Observe first that one can always find a collection $\mathcal{C}$ of alternating cycles satisfying $|\mathcal{C}| / 2 \geq q-p$. Take any $2-$ factor $F$ in $G$. It is a collection of node disjoint cycles which are alternating with respect to a perfect matching. $F$ exists since $G$ is bipartite and $\Delta$-regular. Since $0<q-p \leq n=|F| / 2$ the 2 -factor $F$ satisfies the inequality. In order to minimize the size of $R$ we will have to find a family $\mathcal{C}$ which in addition has a minimum number of edges. Let now $M$ be a perfect matching in $G$.

1. Assume first $p \leq n-|\mathcal{C}| / 2$; then we color in red $p$ edges of $M-\mathcal{C}$ and $q-p$ edges of $M \cap \mathcal{C}$. This is possible since $0<q-p \leq|\mathcal{C}| / 2$. Clearly we will have $|M \cap R|=q$ : by interchanging the edges of $M \cap \mathcal{C}$ and of $\mathcal{C}-M$ we get a perfect matching $M^{\prime}$ with $\left|M^{\prime} \cap R\right|=p$. In such a case $|R|$ has minimum cardinality, since we must have $|R| \geq q$ for any $\mathcal{P}$-feasible $R$. Notice that in this case any $\mathcal{C}$ with $|\mathcal{C}| / 2 \geq q-p$ will do.

2. Suppose now $p>n-|\mathcal{C}| / 2$; we color in red $n-|\mathcal{C}| / 2$ edges of $M-\mathcal{C}$ and we also color $q-(n-|\mathcal{C}| / 2)=q-n+|\mathcal{C}| / 2$ edges of $M \cap \mathcal{C}$ as well as $p-(n-|\mathcal{C}| / 2)=$ $p-n+|\mathcal{C}| / 2$ edges of $\mathcal{C}-M$. This is possible since $0<p-n+|\mathcal{C}| / 2<$ $q-n+|\mathcal{C}| / 2 \leq|\mathcal{C}| / 2$. So we have $|R|=p+q-n+|\mathcal{C}| / 2 \leq n+|\mathcal{C}| / 2$. Again $|M \cap R|=q$ and by interchanging the edges of $M \cap \mathcal{C}$ and of $\mathcal{C}-M$ we get a perfect matching $M^{\prime}$ with $\left|M^{\prime} \cap R\right|=p$.

In order to have a matching $M$ and a matching $M^{\prime}$ having respectively $q$ and $p<q$ red edges, $M^{\prime}$ must be obtained from $M$ by using a collection $\mathcal{C}$ of node disjoint alternating cycles with $|\mathcal{C}| / 2 \geq q-p$ since $|\mathcal{C}|=\left|M \Delta M^{\prime}\right|$; we would otherwise have $0<|M \cap R|-\left|M^{\prime} \cap R\right| \leq|\mathcal{C}| / 2<q-p$. Now for any $\mathcal{P}$-feasible set $R$ which is minimal (inclusionwise), we have two perfect matchings $M, M^{\prime}$ with $|M \cap R|=p,\left|M^{\prime} \cap R\right|=q$. So we have $|R|=p+q-\left|\left(M \cap M^{\prime}\right) \cap R\right| ;|R|$ will be minimum if we maximise the third term. We have $\left|\left(M \cap M^{\prime}\right) \cap R\right| \leq n-|\mathcal{C}| / 2$ where $\mathcal{C}$ is any family of node disjoint alternating cycles with respect to some perfect matching with $|\mathcal{C}| / 2 \geq q-p$; taking such a family $\mathcal{C}$, with $|\mathcal{C}|$ minimum, will give the largest value of $n-|\mathcal{C}| / 2$, so $R$ has minimum cardinality.

Notice that if $p \geq q-2$, we can use a single alternating cycle $C$ instead of the family $\mathcal{C}$ since in any alternating cycle $C$ we have $|C| / 2 \geq 2 \geq q-p$.

Corollary 1. Let $G=(X, Y, E)$ with $|X|=|Y|=n$ be a $\triangle$-regular bipartite graph and let $\mathcal{P}=\{q-a, q\}$ with $1 \leq q \leq n$ and $1 \leq a \leq 2$. The minimum cardinality of $a$ $\mathcal{P}$-feasible set $R$ is given by

$$
|R|=q+\max \{0, q-n+|C| / 2-a\}
$$

where $C$ is a shortest cycle which is alternating with respect to some perfect matching in $G$.

Surprisingly the complexity of finding in a graph $G$ a shortest possible alternating cycle with respect to some maximum matching (not given) is unknown even if 
$G$ is a 3-regular bipartite graph. For reference purposes, this problem will be called the SAC problem (Shortest Alternating Cycle); it is formally defined as follows:

INSTANCE: a graph $G=(V, E)$ and a positive integer $L \leq|V|$

QUESTION: is there a maximum matching $M$ and a cycle $C$ with $|C| \leq L$ and $|C \cap M|=\frac{1}{2}|C|$ ?

Notice that the problem is easy if either a cycle $C$ or a perfect matching $M$ is given.

In order to give a sufficient condition for a regular graph $(X, Y, E)$ with $|X|=$ $|Y|=n$ to have a $\mathcal{P}$-feasible set $R$ with $|R|=n+1$ for $\mathcal{P}=\{0,1, \ldots, n\}$, we will need some preliminaries.

Lemma 1. For any collection of $n$ subsets $S_{1}, \ldots, S_{n}$ of a set $S=\left\{s_{1}, \ldots, s_{n}\right\}$ such that $\left|S_{i}\right|=r \geq \sqrt{n}+1,1 \leq i \leq n$, there exist two subsets $S_{i}, S_{j}$ such that $\left|S_{i} \cap S_{j}\right| \geq 2$.

Proof. Assume we have a collection of $m$ subsets $S_{i}$ of $S$ with $\left|S_{i}\right|=r$ for all $i \leq m$ and $\left|S_{i} \cap S_{j}\right| \leq 1$ for all $i, j \leq m$, then an element $s \in S$ is contained in at most $\frac{n-1}{r-1}$ subsets $S_{i}$ (since the number of subsets $S_{i}$ which contain $s$ and which are otherwise disjoint is $\leq(n-1) /(r-1))$; so the total number $m$ of subsets is at most $\frac{n}{r}\left(\frac{n-1)}{r-1)}\right.$ because we can take $n$ different elements $s$ and in doing this each set is counted $r$ times.

Now if we have $m=n>\frac{n}{r}\left(\frac{n-1)}{(r-1)}\right.$, then there will be two subsets $S_{i}, S_{j}$ with $\left|S_{i} \cap S_{j}\right| \geq 2$. The smallest $r$ verifying $r(r-1) \geq n-1$ is $r=\frac{1}{2}(1+\sqrt{4 n-3})<\sqrt{n}+1$.

In the following, we denote by $\delta(G)$ the minimum degree of a graph $G$ and by $N(x)$ the set of neighbours of $x$. Furthermore $C_{k}$ will denote a cycle of length $k$.

Corollary 2. Let $G=(X, Y, E)$ be a bipartite graph with $|X|=|Y|=n, n \geq 4$. If $\delta(G) \geq \sqrt{n}+1$ then $G$ contains a cycle of length four.

Proof. If $\delta(G) \geq \sqrt{n}+1$, it follows from Lemma 1 that there exist two nodes $x, x^{\prime} \in X$ such that $\left|N(x) \cap N\left(x^{\prime}\right)\right| \geq 2$. Hence, for $y, y^{\prime} \in N(x) \cap N\left(x^{\prime}\right),\left(x, y, x^{\prime}, y^{\prime}\right)$ is a cycle.

Fact 2.1. Let $n, p, q$ be three integers such that $0 \leq q \leq p \leq n$. Then $p+\sqrt{n-p}+1 \geq$ $q+\sqrt{n-q}+1$.

Proof. If $p=q$, the result is obvious. Consider now the case $p>q$. Then $p+$ $\sqrt{n-p}+1 \geq q+\sqrt{n-q}+1$

$$
\begin{aligned}
& \Leftrightarrow p+\sqrt{n-p} \geq q+\sqrt{n-q} \\
& \Leftrightarrow p-q \geq \sqrt{n-q}-\sqrt{n-p} \\
& \Leftrightarrow(p-q)(\sqrt{n-q}+\sqrt{n-p}) \geq p-q \\
& \Leftrightarrow \sqrt{n-q}+\sqrt{n-p} \geq 1
\end{aligned}
$$

This is necessarily true, as $0 \leq q<p \leq n$. 
Fact 2.2. $\frac{n+2\left\lceil\frac{n}{4}\right\rceil+1}{2} \geq 2\left(\left\lceil\frac{n}{4}\right\rceil-1\right)+\sqrt{n-2\left(\left\lceil\frac{n}{4}\right\rceil-1\right)}+1$

$$
\begin{aligned}
& \text { Proof. } \frac{n+2\left\lceil\frac{n}{4}\right\rceil+1}{2} \geq 2\left(\left\lceil\frac{n}{4}\right\rceil-1\right)+\sqrt{n-2\left(\left\lceil\frac{n}{4}\right\rceil-1\right)}+1 \\
& \quad \Leftrightarrow n+2\left\lceil\frac{n}{4}\right\rceil+1 \geq 4\left\lceil\frac{n}{4}\right\rceil-4+2 \sqrt{n-2\left(\left\lceil\frac{n}{4}\right\rceil-1\right)}+2 \\
& \quad \Leftrightarrow n-2\left\lceil\frac{n}{4}\right\rceil+3 \geq 2 \sqrt{n-2\left(\left\lceil\frac{n}{4}\right\rceil-1\right)} \\
& \quad \Leftrightarrow n^{2}+4\left\lceil\frac{n}{4}\right\rceil^{2}+9-4 n\left\lceil\frac{n}{4}\right\rceil-12\left\lceil\frac{n}{4}\right\rceil+6 n \geq 4 n-8\left\lceil\frac{n}{4}\right\rceil+8 \\
& \Leftrightarrow n^{2}+4\left\lceil\frac{n}{4}\right\rceil^{2}+1-4 n\left\lceil\frac{n}{4}\right\rceil-4\left\lceil\frac{n}{4}\right\rceil+2 n \geq 0 \\
& \Leftrightarrow 4\left\lceil\frac{n}{4}\right\rceil \leq 2 n+1+\left(n-2\left\lceil\frac{n}{4}\right\rceil\right)^{2}
\end{aligned}
$$

Notice that $4\left\lceil\frac{n}{4}\right\rceil<n+4 \leq 2 n$ if $n \geq 4$, thus $4\left\lceil\frac{n}{4}\right\rceil \leq 2 n+1+\left(n-2\left\lceil\frac{n}{4}\right\rceil\right)^{2}$.

Lemma 2. Let $G=(X, Y, E)$ be a bipartite simple graph with $|X|=|Y|=n, n \geq 4$. A sufficient condition for $G$ to contain $k$ node disjoint cycles of length four is

$$
\delta(G)-2(k-1) \geq \sqrt{n-2(k-1)}+1
$$

Proof. Let $G_{0}, G_{1}, \ldots, G_{k-1}$ be a sequence of graphs built as follows: $G_{0}=G$ and $G_{i}$ is the subgraph of $G_{i-1}$ obtained by deleting nodes $x, x^{\prime}, y, y^{\prime}$ which form a cycle $C_{4}$.

We have $\delta\left(G_{i}\right) \geq \delta\left(G_{i-1}\right)-2$ and $n_{i}=n_{i-1}-2$ where $n_{i}$ is the number of nodes of the left set (or of the right set) in $G_{i}$.

From fact 2.1, if $1 \leq i \leq k$, we have:

$\delta(G) \geq 2(k-1)+\sqrt{n-2(k-1)}+1 \geq 2(i-1)+\sqrt{n-2(i-1)}+1$. As a consequence we have $\delta\left(G_{i-1}\right) \geq \delta(G)-2(i-1) \geq \sqrt{n-2(i-1)}+1=\sqrt{n_{i-1}}+1$ for all $i(1 \leq i \leq k)$.

So from Corollary $2, G_{i-1}$ contains a $C_{4}$ for all $i(1 \leq i \leq k)$ and by the construction we have found $k$ node disjoint cycles $C_{4}$.

Theorem 2. Let $G=(X, Y, E)$ be a $\triangle$-regular simple bipartite graph with $|X|=$ $|Y|=n \geq 4$ and $\Delta \geq \frac{1}{2}\left(n+2\left\lceil\frac{n}{4}\right\rceil+1\right)$. Let $\mathcal{P}=\{0,1, \ldots, n\}$; then there exists a $\mathcal{P}$-feasible set $R$ with $|R|=n+1$.

Proof. We have $\triangle \geq \frac{1}{2}\left(n+2\left\lceil\frac{n}{4}\right\rceil+1\right) \geq 2\left(\left\lceil\frac{n}{4}\right\rceil-1\right)+\sqrt{n-2\left(\left\lceil\frac{n}{4}\right\rceil-1\right)}+1$ from Fact 2.2. It follows from Lemma 2 that $G$ contains $\lceil n / 4\rceil$ node disjoint cycles $C_{4}$. Let $\left\{x_{2 i+1}, y_{2 i+1}, x_{2 i+2}, y_{2 i+2}\right\}$ be the nodes of cycle $C_{4}^{i}$ for $i=0, \ldots,\lceil n / 4\rceil-1$.

We observe that the number of nodes of $X$ (or of $Y$ ) contained in the cycles $C_{4}^{i}$ is $2\lceil n / 4\rceil \geq n-\lceil n / 2\rceil=\lfloor n / 2\rfloor$.

Let now $H=\left(X^{\prime}, Y^{\prime}, E^{\prime}\right)$ be the subgraph of $G$ obtained by deletion of all the cycles $C_{4}^{i}$ and their nodes. We have $\left|X^{\prime}\right|=\left|Y^{\prime}\right|=n-2\lceil n / 4\rceil \leq\lceil n / 2\rceil$ and $\delta(H) \geq \Delta-2\lceil n / 4\rceil \geq \frac{1}{2}(n+2\lceil n / 4\rceil+1)-2\lceil n / 4\rceil=\frac{1}{2}(n-2\lceil n / 4\rceil+1)=\frac{1}{2}\left(\left|X^{\prime}\right|+1\right)$. 
It is known (see [2] [Corollary 7.3.13]) that such an $H$ is hamiltonian. It has then a 2-factor which can be partitioned into two perfect matchings $M_{H}, M_{H}^{\prime}$ of $H$. Notice that $\left|M_{H}\right|=\left|M_{H}^{\prime}\right|=n-2\lceil n / 4\rceil$.

We now construct $R$ as follows:

$$
R=\left\{\left[x_{i}, y_{i}\right] i=1, \ldots, 2\lceil n / 4\rceil\right\} \cup\left\{\left[x_{1}, y_{2}\right]\right\} \cup M_{H} .
$$

Clearly we construct perfect matchings in $G$ by taking a perfect matching in each $C_{4}^{i}$ and in $H$. Each $C_{4}^{i}(i \geq 1)$ will give matchings with 0 or 2 edges of $R ; C_{4}^{o}$ will give matchings with 1 or 2 edges of $R$. In $H$, the matchings $M_{H}$ and $M_{H}^{\prime}$ have $\left|M_{H}\right|$ or 0 edges in $R$.

From the cycles $C_{4}^{i}$ we can construct matchings having $1,2, \ldots, 2\lceil n / 4\rceil$ edges in $R$. These can be combined with $M_{H^{\prime}}$ to get perfect matchings $M_{i}$ in $G$ having $1,2, \ldots, 2\lceil n / 4\rceil$ edges in $R$. Combining these matchings with $M_{H}$ will give perfect matchings in $G$ having $1+\left|M_{H}\right|, 2+\left|M_{H}\right|, \ldots, 2\lceil n / 4\rceil+\left|M_{H}\right|$ edges in $R$. Since $\left|M_{H}\right|=n-2\lceil n / 4\rceil \leq\lceil n / 2\rceil$ we will produce perfect matchings of $G$ having $i$ edges in $R$ for any $i$ with $1 \leq i \leq n$.

Now since $G$ is regular, we may remove the edges of all cycles $C_{4}^{i}$ and of $M_{H} \cup M_{H}^{\prime}$. We have a $(\triangle-2)$-regular graph, which has a perfect matching $M_{0}$ such that $M_{0} \cap R=$ $\emptyset$. So we have constructed a $\mathcal{P}$-feasible set $R$ with $|R|=2\lceil n / 4\rceil+1+\left|M_{H}\right|=n+1$.

The following is a simple consequence of the König theorem:

Proposition 2. If a 3-regular bipartite graph contains a cycle on four nodes, then this cycle is alternating with respect to some perfect matching.

Remark 1. In general a graph $G$ may not have perfect matchings. We can find a minimum cardinality $\mathcal{P}$-feasible set $R$ for $\mathcal{P}=\{n-1, n\}$ : here $M_{n}$ is a maximum matching which is not perfect; an alternating chain $C=\left\{e_{1}, e_{2}\right\}$ exists which starts at some (exposed) node. We simply remove $e_{2}$ from $M_{n}$ and introduce $e_{1}$ into $M_{n}$ to obtain $M_{n-1}$. So $R=M_{n} \cup\left\{e_{1}\right\}-\left\{e_{2}\right\}$.

Let us mention additional results related to alternating cycles in bipartite graphs.

Theorem 3. Let $G=(X, Y, E)$ be a $\triangle$-regular bipartite graph $(\triangle \geq 3)$ with $|X|=$ $|Y|=n$, then $G$ contains a cycle $C$ with $|C| \leq 2\lceil n / 2\rceil$ which is alternating with respect to some perfect matching.

Proof. Let $\left(M_{1}, M_{2}, \ldots, M_{\triangle}\right)$ be an edge $\triangle$-coloring of $G$; if $M_{1} \cup M_{2}$ is not a hamiltonian cycle, then it contains a cycle $C$ with $|C| \leq 2\lceil n / 2\rceil$. $C$ is clearly alternating for $M_{1}$.

If $M_{1} \cup M_{2}$ is a hamiltonian cycle, then consider any edge $e \in M_{3} ; M_{1} \cup M_{2} \cup\{e\}$ contains 2 cycles using $e$; at least one of them has at most $2\lceil n / 2\rceil$ edges; this cycle is alternating with respect to $M_{1}$ or $M_{2}$. 
Theorem 4. Let $G$ be a $\triangle$-regular bipartite graph (with $\triangle \geq 3$ ) such that for some integral $k \geq 3$ every cycle of length at least $2 k$ has a chord. Then there exists a cycle $C$ with $|C| \leq 2 k-2$ which is alternating with respect to some perfect matching.

Proof. Take a perfect matching $M$ in $G$; since $\Delta \geq 3$ from the König theorem, there exists a perfect matching $M^{\prime}$ with $M^{\prime} \cap M=\emptyset$. Then $M^{\prime} \cup M$ contains a cycle $C$ which is alternating with respect to $M$. Assume $|C| \geq 2 k$; then there is a chord $a b$. It determines with one part of $C$ an alternating cycle $C^{\prime}$ with respect to $M$. Now $\left|C^{\prime}\right| \leq|C|-2$. If $\left|C^{\prime}\right| \geq 2 k$ we continue. We will finally get a cycle $C^{\prime \prime}$ with $\left|C^{\prime \prime}\right| \leq 2 k-2$ which will be alternating with respect to $M$.

A tedious but not difficult enumeration of cases shows the following:

Theorem 5. For a 3-regular bipartite graph $G=(X, Y, E)$ with $|X|=|Y|=n \leq 7$, there exists a set $R \subseteq E$ with $|R| \leq n+2$ which is $\mathcal{P}$-feasible for $\mathcal{P}=\{0,1, \ldots, n\}$.

This result is best possible in the sense that there exists a bipartite 3-regular graph on $2 n=14$ nodes for which the minimum value of $|R|$ is $n+2=9$; this is the so-called Heawood graph (or (3, 6)-cage) (see [8], p.309).

In 3-regular bipartite graphs $G=(X, Y, E)$ with $|X|=|Y|=n \geq 8$ the minimum cardinality of a $\mathcal{P}$-feasible set $R$ for $\mathcal{P}=\{0,1, \ldots, n\}$ is not known.

Finally if we restrict $\mathcal{P}$ to $\{0,1, \ldots, p\}$ with $p \leq 4$, we can state the following:

Theorem 6. Let $p \leq 4$ be an integer. For a 3-regular bipartite graph $G=(X, Y, E)$, with $|X|=|Y|=n \geq 2(p-1)$ there exists a set $R \subseteq E$ with $|R|=p$ which is $\mathcal{P}$-feasible for $\mathcal{P}=\{0,1, \ldots, p\}$.

Proof. We just give the proof for $p=4$; the case $p \leq 3$ can be handled similarly. Let $M, M^{\prime}$ be two disjoint perfect matchings in $G$. So $E-\left(M \cup M^{\prime}\right)$ is also a perfect matching. Suppose $M \cup M^{\prime}$, which is a 2-factor, is connected. It is then a hamiltonian cycle $\mathfrak{C}$ of $G$. Choose two chords of $\mathfrak{C}$, say $[a, b]$ and $[c, d]$, which are at distance at least 2 (i.e. there are no two nodes of the chords that are adjacent). These chords, belonging to $E-\left(M \cup M^{\prime}\right)$, divide the set of edges of cycle $\mathfrak{C}$ into four parts $A, B$, $C$ and $D$.

Let $M_{1}$ (resp. $M_{2}$ ) be the matching containing the chord $[a, b]$ (resp. $[c, d]$ ) and $|M|-1$ edges of $\mathfrak{C}$. Clearly $M_{1}$ and $M_{2}$ use the same edges of $M \cup M^{\prime}$ in two parts, say $A$ and $C$, and different edges of $M \cup M^{\prime}$ in $B$ and $D$. As the chords are at distance at least 2 , there are at least two edges $e_{1}, e_{2} \in M_{1} \cap M_{2}$. Let us distinguish two cases:

1. there exist $e_{1}, e_{2} \in M_{1} \cap M_{2} \cap M$ (if necessary exchange $M$ and $M^{\prime}$ )

It is obvious that there exists an edge $e_{3}$ in $B \cup D$ such that $e_{3} \in M_{1} \cap M$ or $e_{3} \in M_{2} \cap M$ as $M_{1}$ and $M_{2}$ use different edges in $B$ and $D$. Suppose there exists $e_{3} \in M_{1} \cap M$. Take $R=\left\{[a, b], e_{1}, e_{2}, e_{3}\right\}$. Then we have: $|M \cap R|=3$, $\left|M^{\prime} \cap R\right|=0,\left|M_{1} \cap R\right|=4,\left|M_{2} \cap R\right|=2$ and $\left|\left(E-\left(M \cup M^{\prime}\right)\right) \cap R\right|=1$.

2. there exist no two edges $e_{1}, e_{2}$ such that $e_{1}, e_{2} \in M_{1} \cap M_{2} \cap M$ or $e_{1}, e_{2} \in$ $M_{1} \cap M_{2} \cap M^{\prime}$; this of course implies $|A|=|C|=3$. Suppose $e_{1} \in M_{1} \cap M_{2} \cap M$ 
and $e_{2} \in M_{1} \cap M_{2} \cap M^{\prime}$. It is obvious that there exist two edges $e_{3}, e_{4}$ in $B \cup D$ such that $e_{3}, e_{4} \in M_{1} \cap M$ or $e_{3}, e_{4} \in M_{2} \cap M$ as at least one part $B$ or $D$ contains at least four edges. Suppose $e_{3}, e_{4} \in M_{1} \cap M$. Take $R=\left\{e_{1}, e_{2}, e_{3}, e_{4}\right\}$. Then we have: $|M \cap R|=3,\left|M^{\prime} \cap R\right|=1,\left|M_{1} \cap R\right|=4,\left|M_{2} \cap R\right|=2$ and $\left|\left(E-\left(M \cup M^{\prime}\right)\right) \cap R\right|=0$.

Suppose now $M \cup M^{\prime}$ is not connected. Then it consists of at least two cycles. Notice that if there are more than two cycles, the solution is obvious. In fact consider three cycles $C^{1}, C^{2}$ and $C^{3}$. Take $R=\left\{e_{1}, e_{2}, e_{3}, e_{4}\right\}$ such that $e_{1}, e_{2} \in C^{1} \cap M, e_{3} \in C^{2} \cap M$ and $e_{4} \in C^{3} \cap M^{\prime}$. Then of course there exist perfect matchings with $0,1,2,3,4$ edges of $R$. So suppose now that $M \cup M^{\prime}$ consists of exactly two cycles, $C^{1}$ and $C^{2}$. Distinguish two cases:

1. at least one of the cycles, say $C^{1}$, has a chord $[a, b]$

This chord divides cycle $C^{1}$ in two parts $A$ and $B$ which contain both an odd number of edges. Let $M_{1}$ be the perfect matching containing chord $[a, b]$, the edges of $M$ in $A$, the edges of $M^{\prime}$ in $B$ (if necessary exchange $M$ and $M^{\prime}$ ) and edges of $M$ in cycle $C^{2}$. Let $M_{2}$ be the perfect matching containing chord [a,b], the edges of $M_{1}$ in $C^{1}$ and edges of $M^{\prime}$ in cycle $C^{2}$. So there exist at least two edges $e_{1}, e_{2} \in M_{1} \cap M_{2}$ such that $e_{1} \in M$ and $e_{2} \in M^{\prime}$. Consider $e_{3}, e_{4} \in C^{2}$ such that $e_{3}, e_{4} \in M$. Take $R=\left\{e_{1}, e_{2}, e_{3}, e_{4}\right\}$. The we have: $|M \cap R|=3$, $\left|M^{\prime} \cap R\right|=1,\left|M_{1} \cap R\right|=4,\left|M_{2} \cap R\right|=2$ and $\left|\left(E-\left(M \cup M^{\prime}\right)\right) \cap R\right|=0$.

2. none of the two cycles has a chord

This is only possible if both cycles have same length. As $n \geq 6$, we have $\left|C^{1}\right|=$ $\left|C^{2}\right| \geq 6$. Consider two edges $[a, b],[c, d] \in E-\left(M \cup M^{\prime}\right)$ such that nodes $a$ and $c$ are neighbors in $C^{1}$. Let $M_{1}$ be the perfect matching containing [a,b], [c,d], edges of $M$ in $C^{1}$ and edges of $M \cup M^{\prime}$ in $C^{2}$. Let $e_{1}, e_{2} \in C^{1} \cap M_{1}$ and $e_{3} \in C^{2} \cap$ $M_{1} \cap M$. Take $R=\left\{[a, b], e_{1}, e_{2}, e_{3}\right\}$. Then we have: $\left|M_{1} \cap R\right|=4 ;\left|M^{\prime} \cap R\right|=0$, $|M \cap R|=3$, taking $M$ in $C^{1}$ and $M^{\prime}$ in $C^{2}$ we obtain a perfect matching with two edges of $R$, taking $M^{\prime}$ in $C^{1}$ and $M$ in $C^{2}$, we obtain a perfect matching with one edge in $R$.

\section{The Interval Property (IP)}

We shall consider here the special case where $\mathcal{P}$ is a set of consecutive integers and we will characterize graphs which have a property related to such a $\mathcal{P}$. We will exhibit some classes of graphs (bipartite or not) for which a $\mathcal{P}$-feasible set $R$ with minimum cardinality can be obtained in polynomial time. We will denote by $v(G)$ the cardinality of a maximum matching in $G$.

We shall say that $G$ has property IP (interval property) if whenever there are maximum matchings $M_{k}, M_{\nu}$ in $G$ with $\left|M_{k} \cap M_{\nu}\right|=k<v=v(G)$, there are also maximum matchings $M_{i}$ with $\left|M_{i} \cap M_{\nu}\right|=i$ for $i=k, k+1, \ldots, \nu$.

In other words, when $G$ has property IP and there is some $k$ and two maximum matchings $M_{k}, M_{v}$ with $\left|M_{k} \cap M_{v}\right|=k \leq v(G)$, then $R=M_{v}$ is $\mathcal{P}$-feasible for $\mathcal{P}=\{k, k+1, \ldots, v=v(G)\}$ and clearly $R$ has minimum cardinality. 
We define a IP-perfect graph $G$ as a graph in which every partial subgraph has property IP. We recall the reader that a partial subgraph of a graph $G$ is obtained by taking an induced subgraph $G^{\prime}$ and keeping only a subset of edges of $G^{\prime}$.

A cactus is a graph where any two (elementary) cycles have at most one common node. A cactus is odd if all its (elementary) cycles are odd. Notice that a tree is a special (odd) cactus.

Theorem 7. The following statements are equivalent:

(a) $G$ is an odd cactus

(b) $G$ is IP-perfect

Proof. $(b) \Rightarrow(a)$ If $G$ is not an odd cactus, there exists a partial subgraph which is an even cycle $C=M_{v} \cup M_{0}$ with $\left|M_{v}\right|=\left|M_{0}\right| \geq 2$. We have $M_{o} \cap M_{v}=\varnothing$ by construction and $\left|M_{v} \cap M_{v}\right| \geq 2$, but there is no $M_{1}$ with $\left|M_{1}\right|=\left|M_{v}\right|$ and $\left|M_{1} \cap M_{v}\right|=1$.

$(a) \Rightarrow(b)$. Assume we have two maximum matchings $M_{k}, M_{\nu}$ with $\left|M_{k} \cap M_{v}\right|=$ $k<v=v(G)$. Consider $M_{k} \Delta M_{\nu}$. It consists of a collection of node disjoint even alternating chains with total length $2(v-k)>0$.

Starting from $M_{v}$ we may use some of the subchains (by starting from the end nodes saturated by $M_{v}$ ) to replace $r=v-i$ edges of $M_{v}$ by $r$ edges of $M_{k}$ in order to obtain a maximum matching $M_{i}$ with $\left|M_{i} \cap M_{\nu}\right|=i=v-r$ for $i=k+1, \ldots, v(G)$. Since every partial subgraph of $G$ is also an odd cactus, $G$ has property IP.

It follows from Theorem 7 that if we want to find the largest sequence of consecutive integers $\mathcal{P}=\left\{p_{0}, p_{1}, \ldots, p_{s}\right\}$ such that a set $R=M_{\nu}$ is $\mathcal{P}$-feasible for an odd cactus $G$, we have to find in $G$ two maximum matchings $M_{k}, M_{\nu}$ such that $\left|M_{k} \cap M_{\nu}\right|$ is minimum.

Let us examine first the case of bipartite graphs (that include trees but not odd cacti).

Theorem 8. If $G=(X, Y, E)$ is a bipartite graph, there exists a polynomial time algorithm to construct two maximum matchings $M, M^{\prime}$ with a minimum value of $\left|M \cap M^{\prime}\right|$.

Proof. Let us replace each edge $[x, y]$ of $G$ by two $\operatorname{arcs}(x, y)^{0}$ and $(x, y)^{1}$ with capacities $c(x, y)^{0}=c(x, y)^{1}=1$ and costs $k(x, y)^{0}=0, k(x, y)^{1}=1$. Introduce a source $s$ with arcs $(s, x)$ having $c(s, x)=2$ and $k(s, x)=0$ for each node $x$ in X. Similarly for each node $y$ in $Y$ introduce a sink $t$ with $\operatorname{arcs}(y, t)$ with $c(y, t)=2$ and $k(y, t)=0$.

Construct in the network $N$ obtained in this way an (integral) maximum flow $f$ from $s$ to $t$ with minimum cost $K(f)$. Clearly there exists a feasible flow with value $2 v(G)$ (obtained by setting $f(x, y)^{1}=f(x, y)^{0}=1$ for all arcs $(x, y)$ corresponding to the edges $[x, y]$ of a maximum matching in $G$ ). Furthermore no flow can have a value larger than $2 v(G)$ (because this would mean that there is in $G$ a matching $M$ with $|M|>v(G))$. Now the cost of $f$ is equal to the number of $\operatorname{arcs}(x, y)^{1}$ with $f(x, y)^{1}=1$. Since $K(f)$ has been minimized, we have a minimum number of such 
arcs and furthermore $f(x, y)^{1}=1$ implies $f(x, y)^{0}=1$. These are the edges $[x, y]$ of $G$ which are used in both matchings $M, M^{\prime}$. Hence an integral flow $f$ with maximum value $2 v(G)$ and minimum cost $K(f)$ will define two maximum matchings $M, M^{\prime}$ with $\left|M \cap M^{\prime}\right|=K(f)$ minimum.

It is known that such a flow can be constructed in polynomial time (see [1]).

For trees we will present a more efficient algorithm (linear time) in the next section.

Remark 2. For non bipartite graphs, one cannot use the same construction (duplication of edges) and determination of a maximum 2-matching (partial graph $H$ with degrees $d_{H}(z) \leq 2$ for each node $z$ ):

In the graph of Figure 1, we would obtain a 2-matching $H$ consisting of the edges of all four triangles; its cost is 0 . It is clearly not the union of two maximum matchings. The two maximum matchings $M, M^{\prime}$ with $\left|M \cap M^{\prime}\right|$ minimum are $M=M^{\prime}$ given by the heavy edges; the cost of this 2-matching is 6 .

At this stage, we can deduce from Theorems 7 and 8 .

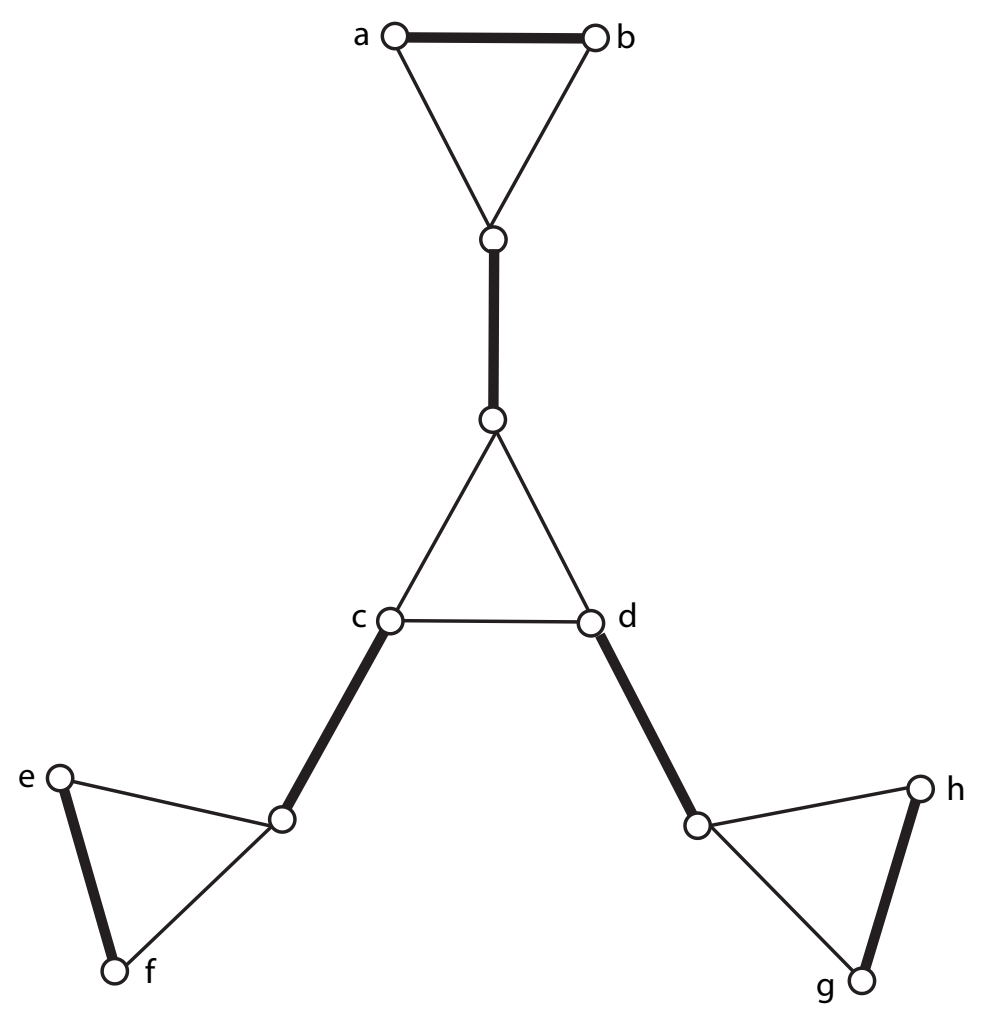

Fig. 1. An odd cactus where the 2-matching algorithm does not give the solution 
Theorem 9. If $G=(V, E)$ is a forest, we can determine in polynomial time a minimum $k$ and a minimum set $R$ of edges to be colored in red in such a way that for $i=k, k+1, \ldots, v(G) G$ has a maximum matching $M_{i}$ with $\left|M_{i} \cap R\right|=i$.

Remark 3. In a graph $G$ with the IP property, there exists a set $R$ with $|R|=v(G)$ such that for $i=0,1, \ldots, v(G) G$ has a maximum matching $M_{i}$ with $\left|M_{i} \cap R\right|=i$ if and only if $G$ has two disjoint maximum matchings.

It should be noticed that finding in a graph two maximum matchings that are as disjoint as possible is NP-complete. This is an immediate consequence of the NP-completeness of deciding whether a 3-regular graph has an edge 3-coloring [10].

We will now show that there is an algorithm to determine if some special odd cacti have two disjoint maximum matchings.

In [6], it is shown that finding a maximum number of edges that can be colored with 2 colors is NP-hard in multigraphs.

D. Hartvigsen has developed (see [9]) an algorithm for constructing in a graph a partial graph $H$ with $d_{H}(v) \leq 2$ for each node $v$, which contains no triangle and which has a maximum number of edges.

Such an algorithm can be used in graphs where the only odd cycles are triangles (these are the so called line-perfect graphs (see [14], [5]). We obtain the following:

Theorem 10. If $G$ is a line-perfect graph, one can determine in polynomial time whether $G$ contains two disjoint maximum matchings.

Proof. We apply the algorithm of D. Hartvigsen that gives a partial graph $H$ with $d_{H}(v) \leq 2$ for each $v$, which contains no triangle and which has a maximum number $|E(H)|$ of edges. Since $G$ has no odd cycle of length 5 or more, $E(H)$ has no odd cycle and is therefore the union of two disjoint matchings $M_{1}, M_{2}$.

We cannot have $|E(H)|>2 v(G)$ because this would imply that $H$ contains a matching $M$ with $|M|>v(G)$. So we have $|E(H)| \leq 2 v(G)$ and if $|E(H)|<2 v(G)$, then clearly $G$ cannot contain two disjoint maximum matchings. So assume we have $|E(H)|=2 v(G)$. Since $\left|M_{1}\right|,\left|M_{2}\right| \leq v(G)$ and $|E(H)|=2 v(G)=\left|M_{1}\right|+\left|M_{2}\right|$, we have two disjoint matchings $M_{1}, M_{2}$ with $\left|M_{1}\right|=\left|M_{2}\right|=v(G)$.

From Theorems 7 and 10 we obtain:

Corollary 3. If $G$ is a cactus where all cycles are triangles, one can determine in polynomial time whether there exists a minimum set $R$ of edges that is $\mathcal{P}$-feasible for $\mathcal{P}=\{0,1, \ldots, v(G)\}$.

It should be noted that in order to find two maximum matchings $M, M^{\prime}$ with $\left|M \cap M^{\prime}\right|$ minimum, we would need to introduce weights on the edges, but apparently this cannot be handled by the above algorithm. 
Since line-perfect graphs may contain even cycles and consequently alternating cycles with respect to some maximum matching, we shall not discuss further about this class (that does not have the IP property) but we shall concentrate on trees.

\section{A Linear Time Algorithm for Trees}

We will describe an algorithm for constructing in a tree $T$ two maximum matchings, say $M$ and $M^{\prime}$, which are as disjoint as possible, i.e. such that $\left|M \cap M^{\prime}\right|$ is minimum. Although such a construction could be performed by network flow technique, we shall sketch here a linear time algorithm.

We will therefore choose a pendent node (a node of degree 1) as a root $r$ and orient all edges towards this root as usual. $T$ becomes an arborescence with arcs $(x, y)$.

We shall start from a leaf (node $w$ with $d_{G}^{-}(w)=0$ ) and follow an oriented path until we meet a node $v$ of indegree $>1$ or the root $r$. Such a node will be called a hip while the paths starting at leaves and reaching $v$ will be called legs. Their parity will be the parity of their number of arcs. Let $e v \geq 0$ (resp. $o d \geq 0$ ) be the number of even (resp. odd) legs starting at hip $v$.

We have several cases to examine:

Case 4.1. $e v=0$, od $\geq 2$

If there are two maximum matchings with minimum intersection, then there are two maximum matchings $M, M^{\prime}$ such that $M \cup M^{\prime}$ contains any two (odd) legs of $v$ and all but the first arc out of $v$ of the remaining (odd) legs. Clearly $M$ and $M^{\prime}$ are disjoint on these legs.

Furthermore the unique $\operatorname{arc}(v, u)$ is not in $M \cup M^{\prime}$ and we can remove it. We construct for the subtree formed by $(v, u)$ and the legs out of $v$ a transversal set $\Im$ consisting of $v$ and all nodes in the legs at even distance from $v$.

Clearly $|\Im|$ is equal to the number of edges of $M\left(\right.$ or $M^{\prime}$ ) in the legs out of $v$.

We remove the subgraph induced by $v$ and the legs out of $v$.

Case 4.2. $e v>0$, od $\geq 2$

We proceed as in case 1 for the arcs on the odd legs and for the even legs, we take the even numbered arcs (starting from the hip $v$ ). Again the transversal set $\Im$ consists of $v$ and all nodes in the legs at even distance from $v$. The edges of the even legs chosen belong to $M \cap M^{\prime}$; we keep track of their number $i(v)$. We can again remove the subgraph induced by $v$ and its legs.

Clearly any two maximum matchings $M_{1}, M_{2}$ in $G$ have $\left|M_{1} \cap M_{2} \cap L(v)\right| \geq i(v)$ where $L(v)$ consists of the unique $\operatorname{arc}(v, u)$ and the legs hanging from hip $v$. Notice that we have $|\mathfrak{\Im}|=|M \cap L(v)|=\left|M^{\prime} \cap L(v)\right|$.

Case 4.3. $e v>0$, od $=1$

We take all even numbered edges of the $e v$ even legs of $v$ and introduce them into $M$ and $M^{\prime}$; let $j(v)$ be their number. We observe that for any two maximum matchings 
$M_{1}, M_{2}$ in $T$ we have $\left|M_{1} \cap M_{2} \cap L^{e}(v)\right| \geq j(v)$ where $L^{e}(v)$ are the even legs at hip $v$.

Then we take for $\Im$ all nodes at odd distance from $v$ in these even legs. We remove all these even legs, so $v$ now has degree 2 (unless it was the root). As before we have $|\mathfrak{s}|=\left|M \cap L^{e}(v)\right|=\left|M^{\prime} \cap L^{e}(v)\right|$.

Case 4.4. $e v>0$, od $=0$

In this case, we take the even numbered edges of the $e v-1$ shortest even legs at hip $v$; let $k(v)$ be their number. We introduce them into $M$ and $M^{\prime}$.

We take for $\Im$ the nodes at odd distance from $v$ in these $e v-1$ even legs. We have $|\Im|=\left|M \cap L^{*}(v)\right|=\left|M^{\prime} \cap L^{*}(v)\right|$ where $L^{*}(v)$ is the collection of edges in these $e v-1$ shortest even legs. Again for any two maximum matchings $M_{1}, M_{2}$ in $T$ we have $\left|M_{1} \cap M_{2} \cap L^{*}(v)\right| \geq k(v)$. We remove $L^{*}(v)$ and $v$ now has degree 2 (unless it was the root).

So we shall start from a leaf of $T$ and apply the above rules until we reach a hip $v$ which is directly connected to the root $r$ of $T$; we will construct matchings $M, M^{\prime}$ and a transversal set $\Im$ such that $|M|=\left|M^{\prime}\right|=\mathfrak{\Im}$. This will be a certificate of optimality for the matchings. Furthermore at each stop the choices (of legs) will have been made in such a way that $\left|M \cap M^{\prime}\right|$ is as small as possible.

It just remains to describe the last step when there is only a single path $P$ left in $T$ from the root $r$. If this leg is even, then we may insert alternately the arcs of $P$ in $M$ and in $M^{\prime}$ and introduce the nodes of $P$ at odd distance from $r$ into $\mathfrak{I}$.

If $P$ is odd, then we have to introduce the odd numbered arcs of $P$ into $M$ and $M^{\prime}$ so $\left|M \cap M^{\prime} \cap P\right|=\lceil|P| / 2\rceil$ and we introduce into $\Im$ the even numbered nodes of $P$.

So we have obtained two maximum matchings $M, M^{\prime}$ in $T$ with a minimum value of $k=\left|M \cap M^{\prime}\right|$ and by coloring the edges of $M$ in red (i.e., setting $R=M$ ) we can construct maximum matchings $M_{i}$ in $T$ with $\left|M_{i} \cap R\right|=i$ for $i=k, \ldots, v(T)$.

\section{Conclusion}

We have examined the problem of finding a minimum subset $R$ of edges for which there exist maximum matchings $M_{i}$ with $\left|M_{i} \cap R\right|=p_{i}$ for some given values of $p_{i}$. Partial results have been obtained for some classes of graphs (regular bipartite graphs, trees, odd cacti with triangles only,...).

In general, our problem requires the determination of a shortest alternating cycle (SAC problem) whose complexity status is open.

Further research is needed to extend our results to other classes of graphs. These problems seem to be more difficult than the spanning tree problems in bicolored graphs mentioned in the introduction; the reason is that it is a special case of three matroid intersection as mentioned in [7]: a matching is an intersection problem of two matroids and the bicoloring $(R, B)$ induces a partition matroid; for trees we simply have, in addition to the partition matroid, a second matroid whose indepen- 
dent sets are the forests in $G$. Such problems are known to be solvable in polynomial time (see [12]).

\section{References}

1. Ahuja, R.K., Magnanti, T.L., Orlin, J.: Network flows. Prentice-Hall, 1993

2. Asratian, A.S., Denley, T.M.J., Häggkvist, R.: Bipartite graphs and their applications. Cambridge University Press, Cambridge, 1998

3. Berge, C.: Graphes. Gauthier-Villars, Paris, 1983

4. Chandrasekaran, R., Kaboadi, S.N., Murty, K.G.: Some NP-complete problems in linear programming. Operations Research Letters, 1, 101-104 (1982)

5. de Werra, D.: On line-perfect graphs. Mathematical programming, 15, 236-238 (1978)

6. Feige, U., Okek, E., Wieder, U.: Approximating maximum edge coloring in multigraphs. Technical Report, Weizmann Institute, 2003

7. Gabor, H.N., Tarjan, R.E.: Efficient algorithms for a family of matroid intersection problems. Journal of Algorithms, 5, 80-131 (1984)

8. Gross, J.L., Yell, J.: Handbook of graph theory. CRC Press, London, 2004

9. Hartvigsen D.: Extensions of matching theory (Ph.D. thesis). Carnegie-Mellon University, 1984

10. Holyer, I.: The NP-completeness of edge-coloring. SIAM Journal on Computing, 10, 718-720 (1981)

11. Karzanov A.V.: Maximum matching of given weight in complete and complete bipartite graphs. Kibernetika, 1:7-11, 1987. English translation in CYBNAW 23, 8-13

12. Lovasz, L., Plummer, M.D.: Matching Theory. Annals of Discrete Mathematics, 29, 1986

13. Garey, M.R., Johnson, D.S.: Computers and Intractability, a Guide to the Theory of NP-Completeness. Freeman, New York, 1979

14. Trotter, L.E.: Line-perfect graphs. Mathematical Programming, 12, 255-259, (1977)

15. Yi, T., Murty, K.G., Spera, C.: Matchings in colored bipartite networks. Discrete Applied Mathematics, 121, 261-277 (2002) 